m Zusammenhang mit der Rettungsaktion der Länder Hamburg und Schleswig-Holstein für die in Schieflage geratene HSH Nordbank wird der Nordstaat wieder diskutiert. Die Zahl der Bundesländer müsse im nächsten Jahrzehnt reduziert werden. Die Neuordnung der Finanzbeziehungen zwischen Bund und Ländern könne dabei nur ein Zwischenschritt sein.

Der größte Teil dieser Neuordnung wurde in der Föderalismuskommission II nicht geschafft, sondern auf die nächste Politikergeneration verschoben. Die Schuldenregelungen sollen erst ab 2020 gelten. Aber zuvor muss noch der bundesstaatliche Finanzausgleich neu geregelt werden, der 2019 endet. Sowohl die SolidarpaktII-Verhandlungen als auch die beiden Reformversuche der Föderalismuskommissionen haben gezeigt, dass Kompromisslösungen zwischen den Ländern schwer zu finden sind. Aber kann man mit einer geringeren Zahl von Verhandlungspartnern schneller zu einem Ergebnis gelangen?

Länderneugliederung kann sowohl bedeuten, die Bundesländer räumlich neu abzugrenzen als auch die Zahl der Bundesländer (durch Fusion) zu verändern. Seit der Wiedervereinigung wurde lediglich 1996 versucht, Berlin und Brandenburg zu vereinigen. Dies scheiterte jedoch in einer Volksabstimmung.

Befürworter von Neugliederungen erhoffen sich durch eine Zusammenlegung Kosteneinsparungen und eine Effizienzerhöhung in Politik und Verwaltung innerhalb der neu geschaffenen Länder. Zudem wird eine deutliche Verringerung des Dauerwahlkampfes in Deutschland erwartet. Außerdem könnte für bisher durch Ländergrenzen zerschnittene Wirtschafts- und Lebensräume (Stadtstaatenproblematik, Halle/ Leipzig, Mannheim/Ludwigshafen etc.) eine nachhaltigere Standortpolitik betrieben werden. Unbestreitbar ist, dass es zu einer Internalisierung

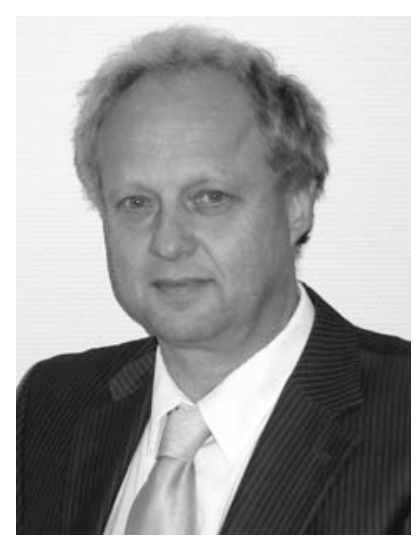

Thomas Lenk

\section{Neugliederung der Bundesländer sinnvoll?}

von grenzüberschreitenden Effekten kommt und die räumliche Allokation der Ressourcen in den betroffenen Regionen effizienter wäre als es die standortpolitischen Entscheidungen in derzeit getrennten Ländern sind.

Ziel sollte es sein, mit einer Neugliederung viele Länder stärker in die Lage zu versetzen, ihre Aufgaben sinnvoll zu erfüllen und die Möglichkeiten zur eigenverantwortlichen Problemlösung zu verbessern. In einem Bundesstaat ist es sinnvoll, Länder mit ähnlicher Wirtschafts- und Finanzkraft zu bilden, da diese dann über ähnliche Kapazitäten zur Aufgabenbewältigung verfügen würden. Auch würde die Ausgleichsintensität bzw. das Ausgleichsvolumen im Länderfinanzausgleich deutlich reduziert werden. Damit wären die Konflikte im Rahmen des bundesstaatlichen Finanzausgleichs wesentlich entschärft. Da aber die wirtschaftlichen Disparitäten nicht durch einen neuen Zuschnitt der Ländergrenzen verschwinden, bedarf es immer eines regionalen Ausgleichs innerhalb der neuen Länder. Hierfür wären dann die Länder zuständig. Für diese regionale Umverteilung dürften politische Mehrheiten leichter zu finden sein, als für eine bundesweite Umverteilung im Rahmen des Länderfinanzausgleichs. Zudem könnte die Abhängigkeit insbesondere kleinerer und finanzschwächerer Länder vom Bund reduziert und damit auch die machtausgleichende Stellung der Länderebene im deutschen föderalen System gestärkt werden.

Gegen eine Neugliederung wirken im gegenwärtigen System des Finanzausgleichs insbesondere das Stadtstaatenprivileg, das diesen pro Einwohner einen um 35\% höheren Finanzbedarf zuspricht, und Sonderbedarfsbundesergänzungszuweisungen, die kleinen Ländern für die politische Führung gewährt werden. Würden diese beiden Regeln nicht mehr angewandt werden, käme es c.p. bei einzelnen Fusionen zu finanzwirtschaftlichen Verlusten im Vergleich zum Status quo.

Auch die Stimmenverteilung im Bundesrat hemmt den Zusammenschluss kleinerer Länder zu einem größeren. So hat derzeit nach Art. 51 Abs. 2 GG z.B. Hamburg drei und Schleswig-Holstein vier Stimmen. Bei einem Zusammenschluss käme es zu einer Schwächung der norddeutschen Position im Bundesrat, da dem neuen Land insgesamt nur vier Stimmen zustehen würden. Daneben gibt es noch viele die Politiker und Bürger betreffende Fragen, wie die nach der (neuen) Landeshauptstadt, landsmannschaftliche Zugehörigkeiten u.a.m.

Zusammenfassend lässt sich festhalten, dass das existierende System der Bund-Länder-(Finanz-) Beziehungen nicht dazu anreizt, Fusionen anzustreben. Eine Länderneugliederung sollte deshalb ernsthaft in Erwägung gezogen werden, wenn die Finanzbeziehungen und die Stimmengewichte im Bundesrat in einer Föderalismuskommission III so geregelt wurden, dass sich für die betroffenen Regionen (ökonomisch) sinnvolle Ergebnisse erzielen lassen. Nur dann können auch die erforderlichen Mehrheiten in den betroffenen Ländern bei den Volksabstimmungen gefunden werden.

Thomas Lenk ist Professor für Finanzwissenschaft an der Universität Leipzig iff_fi wi@wifa.uni-leipzig.de

Wirtschaftsdienst $2009 \cdot 3$ 